\title{
Holographik, the k-essential approach to interactive models with modified holographic Ricci dark energy
}

\author{
Mónica Forte ${ }^{\mathrm{a}}$ \\ Departamento de Física, Facultad de ciencias Exactas y Naturales, Universidad de Buenos Aires, 1428 Buenos Aires, Argentina
}

Received: 19 May 2016 / Accepted: 13 December 2016/ Published online: 24 December 2016

(C) The Author(s) 2016. This article is published with open access at Springerlink.com

\begin{abstract}
We make a scalar representation of interactive models with cold dark matter and modified holographic Ricci dark energy through unified models driven by scalar fields with non-canonical kinetic term. These models are applications of the formalism of exotic k-essences generated by the global description of cosmological models with two interactive fluids in the dark sector and in these cases they correspond to the usual k-essences. The formalism is applied to the cases of constant potential in Friedmann-Robertson-Walker geometries.
\end{abstract}

\section{Introduction}

There are a number of cosmological observations, particularly from Type Ia Supernovae [1-3], Cosmic Microwave Background Radiation [4], and Baryon Accoustic Oscillation $[5,6]$ studies showing an accelerating effect on the expansion of our universe. Therefore, there must be a cosmological component responsible for the repulsive behavior that allows a system to counteract and overcome the gravitational attraction. For this constituent with negative pressure, dubbed dark energy (DE), there have been some proposals. The cosmological constant seems to give the best fit with the observations but also there are good dynamical models including quintessence [7-11], k-essence [12-23], models with internal structure as quintom [24-27] and N-quintom [28] and applications of the holographic principle [29] to cosmology [30-34]. The other majority contribution to the source of Einstein equations is called dark matter (DM), and is the ingredient that comes to supplement the lack of observed non-relativistic matter. Again, we cannot say anything about its nature and moreover, we cannot argue with some symmetry or microphysical criteria that it is evolving regardless of the DE. In fact, the possibility of an interaction between $\mathrm{DM}$ and DE has received many attention in the literature

a e-mail: forte.monica@gmail.com
[35-46] and appears to be even favored over non-interacting cosmologies [47]. This work has the goal of showing the connection between models led by common k-essences (but with special conditions on the signs of the first and second derivatives) and interactive models of cold dark matter (CDM) and modified holographic Ricci type dark energy (MHRDE) fluids. We nickname holographik to these common k-essences to stand out the fact that they are related to interactive models where the dark energy corresponds to a holographic fluid. The idea has precedents in the linking of exotic quintessences $[48,49]$ or exotic k-essences [50] with interactive systems of two arbitrary perfect fluids, but here the purely k-essence $\phi$ is derivable from a Lagrangian of the form $\mathcal{L}=-V_{0} F\left(\dot{\phi}^{2}\right)$ and the interactive systems are compound with fluids whose continuity equation can be replaced by a modified equation using constant coefficients. The MHRDE fluid used here was proposed in [51] as a particular class of the more general holographic Ricci type dark energy introduced in [52] and it was the unique holographic component of a cosmological model that avoided the problem of causality [53]. This statement can be explained as follows. According to the application of the holographic principle to cosmology, the vacuum density of energy can be bounded by the full energy inside a region because it cannot exceed the mass of a black hole of the same size. From effective quantum field theory, an effective infrared (IR) cut-off can saturate the length scale that is included in the expression of the vacuum density of energy and in the literature, the IR cut-off has been taken as the Hubble horizon, or the particle horizon, or the event horizon and also as some generalized IR cut-off. The papers devoted to holographic dark energy models with Hubble horizon or particle horizon as the IR cut-off have shown that these models cannot lead to the current accelerated expansion of the universe. When event horizon is taken as the cut-off, as future event horizon is a global concept of space-time while the density of dark energy is a local quantity, the relation between them will raise challenges to the causality. This leads to the 
introduction of the holographic Ricci type dark energy, where the IR cut-off is taken as proportional to the Ricci scalar curvature, where the problem can be avoided. In the context of interactive systems the MHRDE fluid was used in a plethora of models [54-62].

The paper is organized as follows: In Sect. 2 we consider the non-canonical scalar representation of an interacting cosmological model realized with CDM and a MHRDE fluid and introduce the expressions of the different physical magnitudes in terms of the constant potential $V_{0}$ and of the suitable kinetic functions $F$ of a k-essence field $\phi$. In Sect. 3 we gain deeper insight into the subject analyzing the equation that must be fulfilled by the kinetic functions and the related interactions $Q\left(V_{0}, F\right)$. Also in this section, we show worked examples in both ways. On the one hand, for a given interaction we obtain the corresponding kinetic function and on the other hand we discover which interaction can be considered associated with widely studied k-essences. In Sect. 4 we draw conclusions about the examples in terms of the workability provided by the scalar representation and also on the generation of new functional forms of interaction that can be studied analytically.

\section{The holographic k-essence}

We consider a model consisting of two perfect fluids with an energy-momentum tensor $T_{i k}=T_{i k}^{(1)}+T_{i k}^{(2)}$ where $T_{i k}^{(n)}=\left(\rho_{n}+p_{n}\right) u_{i} u_{k}+p_{n} g_{i k}, \rho_{n}$ and $p_{n}$ being the density of energy and the equilibrium pressure of fluid $n$ and $u_{i}$ their four-velocity. Assuming that the two fluids interact between them in a spatially flat, homogeneous, and isotropic Friedmann-Robertson-Walker (FRW) cosmological background, the Einstein equations reduce to

$$
\begin{aligned}
& 3 H^{2}=\rho_{1}+\rho_{2} \equiv \rho, \\
& \dot{\rho}_{1}+\dot{\rho}_{2}+3 H\left[\left(1+\omega_{1}\right) \rho_{1}+\left(1+\omega_{2}\right) \rho_{2}\right] \\
& \quad=\dot{\rho}+3 H(1+\omega) \rho=0,
\end{aligned}
$$

where $H=\dot{a} / a$ and $a$ stand for the Hubble expansion rate and the scale factor, respectively, and where we consider equations of state $(\operatorname{EoS}) \omega_{i}=\left(p_{i} / \rho_{i}\right)$ for $i=1,2$. Above, we have assumed an overall perfect fluid description with an effective equation of state, $\omega=p / \rho=-2 \dot{H} / 3 H^{2}-1$, where $p=p_{1}+p_{2}$ and $\rho=\rho_{1}+\rho_{2}$. The dot means derivative with respect to the cosmological time and from Eqs. (1) and (2) we get

$$
-2 \dot{H}=\left(1+\omega_{1}\right) \rho_{1}+\left(1+\omega_{2}\right) \rho_{2}=(1+\omega) \rho .
$$

In this paper a more general version of the holographic fluid described in $[51,63]$ is used as DE. This is the simplest case where the density of energy of the DE is expressed as a general function of the Hubble parameter and its derivative, for which the models avoid the causality problem. Then the holographic density of energy $\rho_{2}$ is written as

$\rho_{2}^{\mathrm{MHRDE}}=\frac{2}{A-B}\left(\dot{H}+\frac{3}{2} A H^{2}\right)$,

where $A$ and $B$ are two arbitrary constants that we can suppose that they satisfy $A>B>0$. From (3) and (4) we obtain

$\left(1+\omega_{1}\right) \rho_{1}+\left(1+\omega_{2}\right) \rho_{2}=A \rho_{1}+B \rho_{2}$,

which is a very useful relation because in our description of the interactive system the equations of state must be constant and in general this does not happen with the EoS of the holographic fluid. Note also that Eqs. (1), (2), and (5) allow us to write the partial densities of energy as

$\rho_{1}=-\frac{B \rho+\rho^{\prime}}{A-B} \quad \rho_{2}=\frac{A \rho+\rho^{\prime}}{A-B}$,

for $\rho^{\prime}=\dot{\rho} / 3 H$.

The interaction $Q$ that connects both fluids is specified through the partial equations of conservation,

$\dot{\rho}_{1}+3 H\left(1+\omega_{1}\right) \rho_{1}=-3 H Q$,

$\dot{\rho}_{2}+3 H\left(1+\omega_{2}\right) \rho_{2}=3 H Q$.

Or better, a modified interaction $Q_{\mathrm{M}}$ can be defined by using Eqs. (5) and (7) by means of

$\dot{\rho}_{1}+3 H A \rho_{1}=-3 H Q_{\mathrm{M}}$,

$\dot{\rho}_{2}+3 H B \rho_{2}=3 H Q_{\mathrm{M}}$.

Clearly, the relation between $Q_{\mathrm{M}}$ and $Q$ is $Q_{\mathrm{M}}=Q+(1-$ A) $\rho_{1}=Q+\left(B-\omega_{2}-1\right) \rho_{2}$, where we apply the formalism to interactions between cold dark matter (CDM) and a modified holographic Ricci type dark energy (MHRDE) fluid.

Now, as was done with the exotic canonical scalar field in [48] and with the exotic field in [50], we propose that the interactive system as a whole be represented by a unified model driven by a special class of purely k-essence field $\phi$ (labeled by a constant potential $V_{0}$ and a kinetic function $\left.F(x), x=-\dot{\phi}^{2}\right)$, through the relationship

$(1+\omega) \rho=A \rho_{1}+B \rho_{2}=-2 V_{0} x F_{x}(x), \quad F_{x}=\frac{\mathrm{d} F(x)}{\mathrm{d} x}$.

Then the global density of energy $\rho$ and the global pressure $p=\omega \rho$ can be written as

$\rho=V_{0}\left(F(x)-2 x F_{x}(x)\right), \quad p=-V_{0} F(x)$.

The field $\phi$ satisfies the equation of movement

$\left[F_{x}+2 x F_{x x}\right] \ddot{\phi}+3 H F_{x} \dot{\phi}=0 \quad F_{x x}=\mathrm{d} F_{x} / \mathrm{d} x$, 
which allows us to find the functional form of the k-field $\phi$ once the kinetic function $F(x)$ is given. If the kinetic function is strictly monotonic $F_{x} \neq 0$, there is the well-known first integral

$\sqrt{-x} F_{x}=m_{0} a^{-3}$

for $m_{0}$ a constant of integration. Alternatively, when the kinetic functions have an extreme $x_{e}=x\left(t_{e}\right)$ such that $F_{x}\left(x_{e}\right)=0$, the above first integral (12) does not exist. Instead, at time $t=t_{e}$, Eq. (11) is reduced to $\left.x_{e} F_{x x}\left(x_{e}\right) \ddot{\phi}\right|_{t_{e}}=$ 0 and thus it must happen that $\dot{\phi}$ has a root or an extreme at $t=t_{e}$, or that $F(x)$ has a saddle point at $x_{e}$. We will not address cases with non-monotonic kinetic functions.

We must note that, from (4), (6), (10), and (11), the partial densities of energy are

$$
\begin{aligned}
\rho_{1} & =-\frac{V_{0}}{A-B}\left(B F(x)-2 x F_{x}(x)(B-1)\right), \\
\rho_{2}^{\mathrm{MHRDE}} & =\frac{V_{0}}{A-B}\left(A F(x)-2 x F_{x}(x)(A-1)\right) \\
& =\frac{A-1-\omega}{A-B} \rho,
\end{aligned}
$$

and therefore, with $A-B>0$ holding, the maximum possible value for the overall EoS should be $\omega=\omega_{\max }=A-1$. This one is the first characteristic that these "special" kessences must have and, interestingly, it comes exclusively from the associated interactive models using MHRDE fluids because $\rho_{2}^{\mathrm{MHRDE}}$ must be non-negative. The expression for the global equation of state $\omega$ in the unified representation of the $\mathrm{k}$-essence is

$\omega=-\frac{F}{F-2 x F_{x}}$

and so (13b) and (14) imply $-2 x F_{x} /\left(F-2 x F_{x}\right) \leq A$.

Also, from (3) and (5) is $\omega=(A-1+(B-1) r) /(1+r)$, where $r=\rho_{2} / \rho_{1}$. Thus, if the universe supports a constant $\operatorname{EoS} \omega=\omega_{0}$, then the ratio between densities must be a constant $r=r_{0}=\left(A-1-\omega_{0}\right) /\left(1+\omega_{0}-B\right)$. Conversely, in these models with an interactive MHRDE fluid, we cannot have a stationary solution to the problem of coincidence, $r=r_{0}$, without paying the price of a universe with constant EoS $\omega_{0}$. In that sense, from (14) we can see that the polynomial kinetic functions $F=(-x)^{n}$, with $n=$ constant, have constant $\omega=(2 n-1)^{-1}$. Therefore the interactive models with interactions associated with these $F$ should not be considered interesting examples to describe realistic cosmological models.

Figure 1 describes the global $\operatorname{EoS} \omega=g /(1-g)$ in terms of the auxiliary function $g \equiv F /\left(2 x F_{x}\right)$ and also shows the prohibited zone $\omega \geq A-1$. There, the left branch $(g<1-1 / A)$ correctly describes a unified model of which the behavior interpolates between a stiff $[64,65]$, radiation or $\omega$

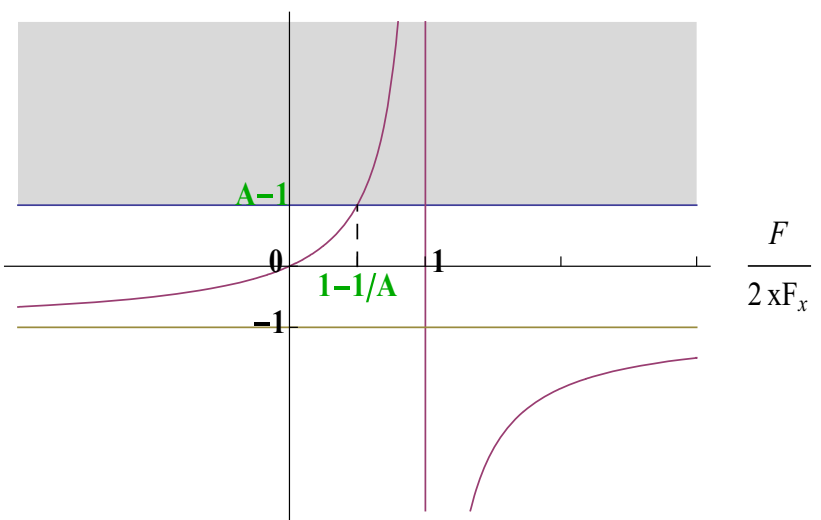

Fig. 1 Evolution of the global equation of state for the holographic unified model as a function of the magnitude $g=F /\left(2 x F_{x}\right)$. The shaded area corresponds to the prohibited values $\omega \geq A-1$, throughout all the evolution of the model. The maximum $\omega_{M}=A-1$ is reached at $g=(A-1) / A$ belonging to the left branch of the graph, the more useful in modeling realistic universes. The right branch is related with phantom universes. The models with asymptotic stiff behavior must have $g \leq 1 / 2$ and those with asymptotic radiation behavior must have $g \leq 1 / 4$. The models with asymptotic dust behavior must have $g \leq 0$ and $F \geq 0$

dust type (for $A=2,4 / 3$ or 1 ) and a cosmological constant type. The right branch $(g>1)$ describes phantom models provided that the EoS is kept $\omega<-1$ along the whole cosmological history.

Let us focus on left branch. The bound $\omega \leq A-1$ results in the bound $g \leq 1-1 / A$ and therefore in the "bounding" functions $F(x)_{\max }=F_{0}(-x)^{A /(2(A-1))}$ for $A=2$ or $A=$ $4 / 3$ and anyone for which $g<0$ if $A=1$. The meaning of "bounding" is evident in Fig. 2, where the general behaviors of $\omega(x)$ for different functions $F$ appear "limited" by the curve with $n=0$

Other two conditions exist to carry out for these functions, which come from the reality of the Hubble factor $H$ and from the stability of the model. From (10) the total density of energy can be written as $\rho / V_{0}=2 x F_{x}(g-1)$. and with positive potentials and $g<1$ it must always be observed that $F_{x}>0$. Therefore, this second condition leads to $F<0$ for $0<g<1-1 / A$ and to $F>0$ for $g<0$.

The last restriction arises from having considered the adiabatic speed of sound $c_{\mathrm{s}}^{2}=(\delta p / \delta \rho)_{\mathrm{s}}=p_{x} / \rho_{x}$ (the subscript $s$ means at constant entropy), because the local stability and causality requirements $0 \leq c_{\mathrm{s}}^{2} \leq 1$ [66-72] determine, through $c_{\mathrm{s}}^{2}=F_{x} /\left(F_{x}+2 x F_{x x}\right)$, the realistic models to be those with $F_{x x} \leq 0$. We use this last condition although in [73] it is shown that the condition $c_{\mathrm{s}}^{2} \leq 1$ is not necessary for causality.

All three conditions: $g<1, F_{x}>0$, and $F_{x x} \leq 0$, are essential to describe realistic models driven by k-essence that are associated with acceptable interactions $Q$ in the dark sector. 


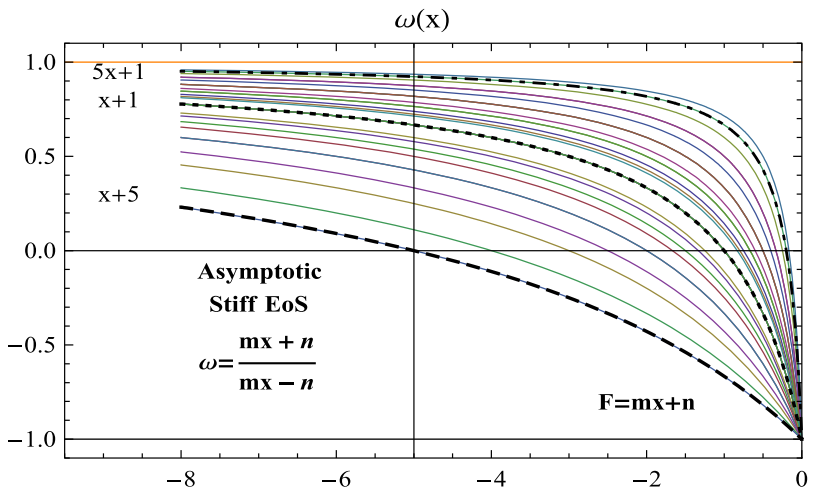

$\omega(\mathrm{x})$

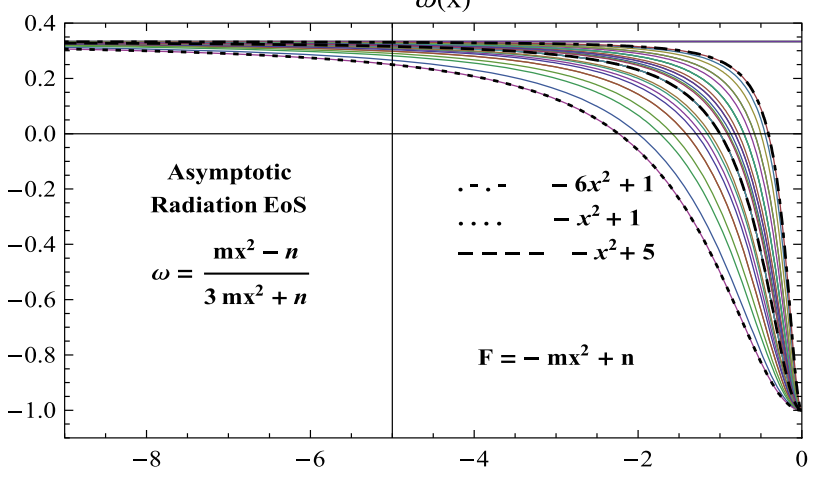

$\omega(\mathrm{x})$

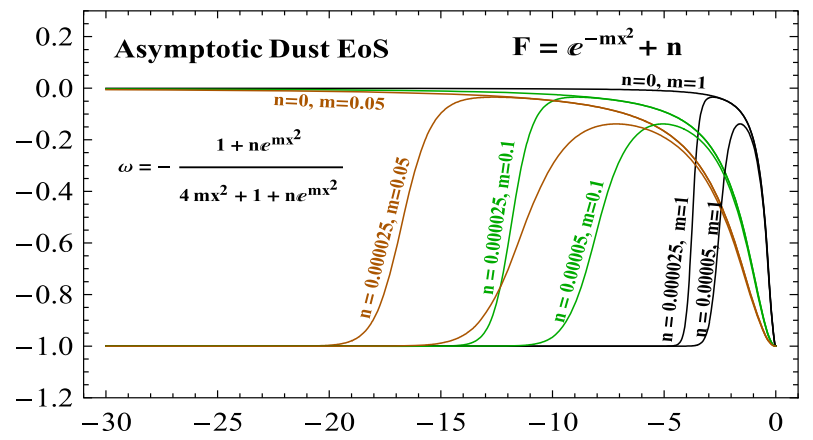

Fig. 2 Top panel EoS interpolating between $\omega=1$ and $\omega=-1$ corresponding to the kinetic functions $F[x]=-m x+n, m=$ $1,2,3,4,5,6, n=1,2,3,4$, 5. Intermediate panel EoS interpolating between $\omega=1 / 3$ and $\omega=-1$ corresponding to the kinetic functions $F[x]=-m x^{2}+n, m=1,2,3,4,5,6, n=1,2,3,4,5$. Bottom panel EoS interpolating between $\omega=0$ and $\omega=-1$ or without dust like era but with an accelerated behavior at early time for tiny $n$, corresponding to the kinetic functions $F(x)=e^{-m x^{2}}+n$, with $m=1,0.1,0.05$, $n=0,0.00005,0.05$

There are several functions that satisfy these three conditions. For example the quadratic function $F[x]=-m x^{2}+$ $n x+c$, which includes the linear one, the one proportional to the tachyonic function $F[x]=m \sqrt{1+x}+n$, the exponential $F(x)=e^{-m x^{2}}+n$ and also $F(x)=-m \cosh (\sqrt{-x})$ with $m>0, n>0$, and $c>0$. Some of them will be used in the next section to find the appropriate associated interaction $Q$ in the dark sector. Figure 2 shows the EoS corresponding to functions $F(x)_{\text {stiff }}=-m x+n, F(x)_{\mathrm{rad}}=-m x^{2}+n$ and
$F(x)_{\text {dust }}=e^{-m x^{2}}+n$, with $m>0$ and $n>0$, for which we can see the corresponding asymptotic limits.

Note that the global equation of state of the k-essence is independent of the potential used and therefore the above results preserve their validity for variable forms of $V$, but in the last cases the first integral (12) no longer exists. Moreover, note that the crossing of the phantom divide line (PDL) is not allowed. This was for the first time proven for k-essence in [74].

\section{The associate interactions}

The results of the previous section are quite general and apply to any kinetic function $F(x)$, but the particular choice of the function will be determined by the interaction $Q$ that manages the evolution of both fluids. Equations (8b) and (14) let us write the equation that must be fulfilled by the kinetic function $F(x)$ once the interaction $Q_{\mathrm{M}}\left(V_{0}, F\right)$ is fixed,

$$
\begin{gathered}
\left(\frac{Q_{\mathrm{M}}}{V_{0}}-B \frac{\left[A F-2 x(A-1) F_{x}\right]}{(A-B)}\right)(2 M-(A-B) N) \\
\quad+2 x F_{x} A N=0,
\end{gathered}
$$

with $M=F_{x}+x F_{x x}$ and $N=\left((2-A) F_{x}-2 x F_{x x}(A-\right.$ 1)) $/(A-B)$.

The expression $Q_{\mathrm{M}}\left(V_{0}, F\right)$ means that the interaction, often expressed as a function of $\rho$ and its derivatives, should be given using Eqs. (6), (10), and (12), and $\rho^{\prime}=2 x F(x) V_{0}$.

Equation (15) is a highly nonlinear equation for $F$. However, the change of variables $\zeta=\int \rho_{x} /\left(2 x F_{x} V_{0}\right) \mathrm{d} x$ and $\rho(x)=V_{0}\left(F-2 x F_{x}\right)$ lets us obtain a simpler differential equation for $\rho$,

$\rho^{\prime \prime}+(A+B) \rho^{\prime}+A B \rho=Q_{\mathrm{M}}(A-B)$,

with $\rho^{\prime}=\mathrm{d} \rho / \mathrm{d} \zeta$ and $\rho^{\prime \prime}=\mathrm{d}^{2} \rho / \mathrm{d} \zeta^{2}$. This is the holographic version [54] of the already known source equation for the energy density described in [75]. On the other hand, Eq. (15) allows one to use the representation in both directions. One direction is to find the system handled by the k-essence $F$ that represents the interactive system and the other one is to assign an interaction $Q$ to an interactive system that is studied as a unified model of k-essence. Let us have a look at some worked examples.

\section{- Examples $Q \rightarrow F$ \\ - CDM and MHRDE}

This interesting case was already presented at the general formalism developed in [50], where it was applied to the null interaction $Q=0$ or equivalently when we replace $Q_{\mathrm{M}}=(1-A) \rho_{1}$ in (15). The solution is $F(x)=\left(F_{0}+F_{1} \sqrt{-x}\right)^{B /(B-1)}$, with $F_{0}<0$, $F_{1}>0,0<B<1$, and lets us write the den- 
sities of energy $\rho^{\mathrm{MHRDE}}=b_{1} a^{-3}+b_{2} a^{-3 B}$ and $\rho_{2}^{\mathrm{MHRDE}}=((A-1) /(A-B)) b_{1} a^{-3}+b_{2} a^{-3 B}$. It can be seen that the MHRDE fluid is always a selfinteracting component, because even when $Q$ is null, the dark energy component is far from remaining independent of the CDM. The asymptotic values of the $\operatorname{EoS} \omega=-b_{2}(1-B) a^{3(1-B)} /\left(b_{1}+b_{2} a^{3(1-B)}\right)$ are 0 and $B-1<A-1$ in the asymptotic limits $a \rightarrow 0$ and $a \rightarrow \infty$, respectively. However, the model is not viable because always $c_{\mathrm{s}}^{2}<0$.

\section{- The holographic $\Lambda$}

In this example we consider the case in which a holographic interactive fluid is behind the concept of cosmological constant. The system of a CDM fluid interacting with a MHRDE fluid (5) through the interaction $\Delta Q=B \rho+(1-\Delta) \rho^{\prime}, \Delta=A-B$, can be interpreted as a cosmological model driven by a purely k-essence identified by the constant potential $V_{0}$ and the kinetic function obtained from (15), $F=F_{1}+2 F_{0} \frac{(1-A)}{A}(-x)^{\frac{A}{2(A-1)}}$, with the positive constants of integration $F_{0}$ and $F_{1}$. From (10), (12), and (13b) the expressions for the global density of energy and the density of energy of MHRDE fluid are

$\rho=\Lambda \frac{A-B}{A}+\frac{\rho_{\mathrm{m}}}{a^{3 A}} \quad \rho_{2}=\Lambda$,

respectively, with $\Lambda=A V_{0} F_{1} /(A-B)$ and $\rho_{\mathrm{m}}=$ $2 V_{0} m_{0}^{A} /\left(A F_{0}^{A-1}\right)$.

If $1<A<2$, the corresponding global EoS,

$\omega=-\frac{\Lambda(A-B)+A(1-A) \rho_{\mathrm{m}} a^{-3 A}}{\Lambda(A-B)+A \rho_{\mathrm{m}} a^{-3 A}}$,

ranges between the values $\omega_{\mathrm{et}}=A-1$ at early times and $\omega_{l t}=-1$ at late times and the sound speed is $c_{\mathrm{s}}^{2}=A-1<1$.

Solving $\rho_{2}^{\mathrm{MHRDE}}=\left(2 \dot{H}+3 A H^{2}\right) /(A-B)=\Lambda$ we obtain the factor of scale

$$
\begin{aligned}
a(t) & =\left(\cosh \left(\kappa\left(t-t_{0}\right)\right)+H_{0} \sinh \left(\kappa\left(t-t_{0}\right)\right)\right)^{\frac{2}{3 A}} \\
\kappa^{2} & =3 A \Lambda(A-B) / 4,
\end{aligned}
$$

where we set $t_{0}$ as the present time for which the factor of scale is $a\left(t_{0}\right)=1$ and the Hubble parameter is $H\left(t_{0}\right)=H_{0}$. Notice that the argument of the hyperbolic functions in (19) corresponds to the usual solution of the factor of scale for the model $\Lambda \mathrm{CDM}$ if $B=\left(A^{2}-1\right) / A$. Also, Eq. (17) corresponds to the model $\Lambda$ plus WDM ( $A$ is very slightly greater than one) or to the model $\Lambda$ plus radiation $(A=4 / 3)$. However, unlike a true cosmo- logical constant, the equation of state for dark energy $\omega_{2}=\omega \rho / \rho_{2}$ diverges at early times and tends asymptotically to $-\Delta / A$ at late times, because its expression is

$\omega_{2}=-\frac{(A-B)}{A}+\frac{(A-1) \rho_{\mathrm{m}}}{\Lambda a^{3 A}}$.

\section{- The sign-change holographic}

There exist a number of works that studied interactions able to change their sign along the evolution of the universe. One of them is $Q_{\mathrm{sc}}=B \rho_{2}-\rho_{1}$, which replaced in (15) allows us to obtain two linear differential equations $x F_{x}-y^{ \pm} F=0$ where $y^{ \pm}=\sqrt{A B} /(2(\sqrt{A B} \pm 1))$ and then the two kinetic functions $F^{ \pm}=F_{0}^{ \pm}(-x)^{y^{ \pm}}$. Using the first integral (12) for each particular kinetic function, the corresponding global energy density is

$$
\begin{aligned}
& \rho=\left(\rho_{0}-\rho_{-}\right) a^{3 \sqrt{A B}}+\rho_{-} a^{-3 \sqrt{A B}}, \\
& \rho_{-}=\frac{V_{0} F_{0}^{-}}{(1-\sqrt{A B})},
\end{aligned}
$$

with $\rho_{0}$ the actual global density and the constant of integration $m_{0}^{-}$coming from (12) taken so that $m_{0}^{-}=-F_{0}^{-} y^{-}$. Therefore, the global EoS oscillates between $-(1-\sqrt{A B})$ at early times and $-(1+\sqrt{A B})$ at late times, as can be seen in

$$
\omega=-\frac{\left(\rho_{0}-\rho_{-}\right)(1+\sqrt{A B}) a^{6 \sqrt{A B}}+\rho_{-}(1-\sqrt{A B})}{\left(\rho_{0}-\rho_{-}\right) a^{6 \sqrt{A B}}+\rho_{-}} .
$$

Assuming $\Delta>0$ and $\sqrt{A B}<1$, the change of sign of the interaction is produced at $\omega_{\mathrm{sc}}=(A B-$ 1) $/(B+1)$ for which the factor of scale is

$$
a_{\mathrm{sc}}=\left(\frac{\rho_{-}(\sqrt{A B}(B+1)-(A+1) B)}{\left(\rho_{0}-\rho_{-}\right)(\sqrt{A B}(B+1)+(A+1) B)}\right)^{\frac{1}{6 \sqrt{A B}}} .
$$

This interactive system, affected by $Q_{\mathrm{sc}}$ is consistently maintained until $\rho_{1}$ is exhausted at

$a_{\max }=\left(\frac{\rho_{-}(\sqrt{A B}-B)}{\left(\rho_{0}-\rho_{-}\right)(\sqrt{A B}+B)}\right)^{\frac{1}{6 \sqrt{A B}}}$,

when the sign change has already occurred because $a_{\max }>a_{\text {sc }}$.

Figure 3 shows the global density of energy (21) and the partial densities of energy

$\rho_{1}=\frac{1}{\Delta}\left\{-\left(\rho_{0}-\rho_{-}\right)(\sqrt{A B}+B) a^{3 \sqrt{A B}}+\rho_{-}(\sqrt{A B}-B) a^{-3 \sqrt{A B}}\right\}$, 


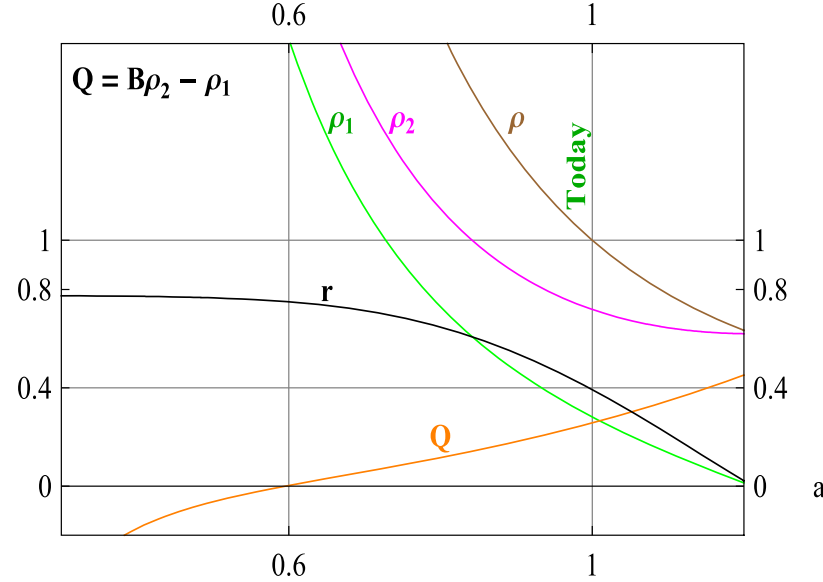

Fig. 3 Densities of energy, ratio $r=\rho_{1} / \rho_{2}$ and $Q_{\text {sc }}$ for $\rho_{0}-\rho_{-}=$ $0.04, \rho_{-}=0.96, A=5 / 4, B=3 / 4$

$\rho_{2}=\frac{1}{\Delta}\left\{\left(\rho_{0}-\rho_{-}\right)(A+\sqrt{A B}) a^{3 \sqrt{A B}}+\rho_{-}(A-\sqrt{A B}) a^{-3 \sqrt{A B}}\right\}$,

where it can be seen that with the right choice of the constants of integration $\left(\rho_{0}-\rho_{-}\right)$and $\left(\rho_{-}\right)$, the model is consistent with current estimates of dark energy densities and shows a relief in the problem of coincidence. Also, the ratio $r=\rho_{1} / \rho_{2}$ and $Q_{\text {sc }}$ are depicted for $\rho_{0}-\rho_{-}=0.04, \rho_{-}=0.96, A=5 / 4$, $B=3 / 4$.

\section{- Examples $F \rightarrow Q$}

- The linear function $F(x)=1+m x$, with $m>0$, was already used in $[15,21]$ with exponential potentials. Here, with the constant $V=V_{0}$, it is replaced in (15) obtaining the interaction $Q_{\mathrm{M}} \Delta=A B \rho+(A+$ $B-2) \rho^{\prime}$. That is, a cosmological model with CDM fluid interacting with MHRDE through the interaction $\Delta Q=B \rho+(B-1) \rho^{\prime}$ can be seen as a model driven by a purely linear k-essence. With the greatest simplicity, the densities of energy and the EoS of the global model are obtained as well as the time variation of the scale of factor and the k-essence field $\phi$. We have

$$
\begin{aligned}
& \rho=V_{0}+\frac{\rho^{0}}{a^{6}}, \quad \rho_{2}=\frac{1}{\Delta}\left(A V_{0}+(A-1) \frac{\rho^{0}}{a^{6}}\right), \\
& a(t)=\left[\frac{\sinh \left(\sqrt{3 V_{0}} t\right)}{\sinh \left(\sqrt{3 V_{0}} t_{\mathrm{T}}\right)}\right]^{1 / 3} \quad \rho^{0}=\frac{m_{0}^{2} V_{0}}{m}, \\
& \omega=-\frac{V_{0} a^{6}-\rho^{0}}{V_{0} a^{6}+\rho^{0}}, \quad(26 \mathrm{~b}) \\
& \phi=\phi_{0} \ln \left(\tanh \left(\frac{\sqrt{3 V_{0}} t}{2}\right)\right) \quad \phi_{0}=\frac{m_{0} \sinh \left(\sqrt{3 V_{0}} t_{\mathrm{T}}\right)}{m \sqrt{3 V_{0}}},
\end{aligned}
$$

with $t_{\mathrm{T}}$ the actual time. The adiabatic velocity of sound is constantly equal to 1 as in the cases of the quintessence regardless of the values of the parameters $A$ and $B$.

- In [20], the simple quadratic function $F(x)=\frac{b}{6}+$ $x-\frac{x^{2}}{2 b}$ is used with the arbitrary parameter $b>0$ to ensure positivity of the density of energy and stability observed through the speed of sound. In this context of purely holographic k-essence with constant potential $V_{0}$ it leads, through (15), to the associated interaction $Q_{\mathrm{M}} \Delta=A B \rho+(A+B-1) \rho^{\prime}+\rho^{\prime \prime} / 4+$ $\rho^{\prime} /\left(2 \sqrt{6 \rho / b V_{0}}\right)$ or

$Q \Delta=B \rho+B \rho^{\prime}+\frac{\rho^{\prime \prime}}{4}+\frac{\rho^{\prime}}{2 \sqrt{\frac{6 \rho}{b V_{0}}}}$.

From (12) we obtain the algebraic equation $h^{3}+h-$ $u=0$ for $h=\sqrt{-x / b}$ and $u=m_{0} b^{-1 / 2} a^{-3}$, whose unique real solution allows us to write the first integral $\dot{\phi}=\sqrt{-x}$ for the kinetic function as

$$
\begin{aligned}
\sqrt{\frac{-x}{b}}= & -\frac{(2 / 3)^{1 / 3} b^{1 / 6} a}{\left(9+\sqrt{3} \sqrt{4 b a^{6}+27}\right)^{1 / 3}} \\
& +\frac{\left(9+\sqrt{3} \sqrt{4 b a^{6}+27}\right)^{1 / 3}}{2^{1 / 3} 3^{2 / 3} b^{1 / 6} a},
\end{aligned}
$$

and thus, from (10) and (12), the global energy density $\rho=\frac{3 V_{0} b}{2}(1 / 3-x / b)^{2}$ proves to be

$$
\begin{aligned}
\rho=\frac{3 V_{0} b}{2}\left[\frac{1}{3}+\right. & -\frac{(2 / 3)^{1 / 3} b^{1 / 6} a}{\left(9+\sqrt{3} \sqrt{4 b a^{6}+27}\right)^{1 / 3}} \\
& \left.+\frac{\left(9+\sqrt{3} \sqrt{4 b a^{6}+27}\right)^{1 / 3}}{2^{1 / 3} 3^{2 / 3} b^{1 / 6} a}\right)^{2},
\end{aligned}
$$

where the constant of integration in (12) is taken as $m_{0}=1$. The global EoS (14),

$\omega=-\frac{1}{3} \frac{\left(-\frac{x}{b}+1+\frac{2}{\sqrt{3}}\right)\left(\frac{x}{b}-1+\frac{2}{\sqrt{3}}\right)}{\left(-\frac{x}{b}+1 / 3\right)^{2}}$,

shows that this interactive model exhibits a dust type behavior $\omega=0$ when the time evolution of the kessence is $\dot{\phi}=\sqrt{-x_{\text {root }}}=\sqrt{b(-1+2 / \sqrt{3})}$, that is, when $a_{\text {dust }}=1.3 b^{-1 / 6}$. Figure 4 shows that the dust behavior of the global EoS can be accommodated by varying the parameter $b$, and also it shows that the EoS has a single maximum regardless of $b$, corresponding to $A=3 / 2$. 


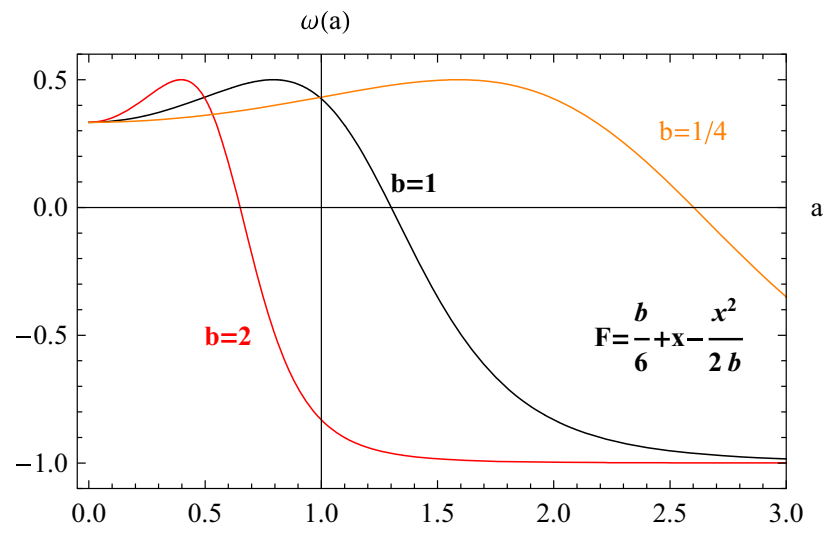

Fig. 4 Global equation of state for the quadratic function $F(x)=$ $\frac{b}{6}+x-\frac{x^{2}}{2 b}$ for different parameters $b$. It can be seen that the maximum is independent of $b$

Thus, the constant $A$ is fixed for the kinetic function and $b$ is fixed by the astronomical data. The remaining constants $V_{0}$ and $B$ are determined by the current overall energy of density $\rho(a=1)$ and by the ratio between dark densities of energy $r=\rho_{1} / \rho_{2}=$ $-\left(B\left(F-2 x F_{x}\right)+2 x F_{x}\right) /\left(A F-2 x F_{x}(A-1)\right)$ at its present value $r(a=1)$, respectively.

The adiabatic velocity of sound $c_{\mathrm{s}}^{2}=F_{x} /\left(F_{x}+\right.$ $\left.2 x F_{x} x\right)=(b-x) /(b-3 x)$ oscillates between $\left.c_{s}^{2}\right|_{\text {et }}=0$ at early time and $\left.c_{\mathrm{s}}^{2}\right|_{l t}=1 / 3$ at late times.

- The kinetic function $F=\alpha-\beta \cosh (\sqrt{-x})$ with $\alpha>\beta>0$ meets the requirement $F_{x}=$ $\beta \sinh (\sqrt{-x}) /(2 \sqrt{-x})>0$ in order that the density of energy is always positive and $F_{x x}=-(\beta \cosh$ $(\sqrt{-x}) /\left(4(-x)^{3 / 2}\right)(\sqrt{-x}-\tanh (\sqrt{-x}))<0$ for the global model is stable. Then, using (12), we find that $\sqrt{-x}=\sinh ^{-1}\left(a^{-3}\right)$ if the constant of integration is taken as $m_{0}=\beta / 2$ and thus the global density of energy is

$\rho=V_{0}\left(\alpha-\frac{\beta}{a^{3}} \sqrt{1+a^{6}}+\frac{\beta}{a^{3}} \sinh ^{-1}\left(a^{-3}\right)\right)$.

Equation (31) can be considered as the global density of energy of an interactive cosmological system filled with CDM and MHRDE that, affected by the interaction $Q$,

$$
\begin{aligned}
\Delta Q= & B\left(\rho+\rho^{\prime}\right) \\
& +\beta V_{0}\left[\frac{\alpha V_{0}-\left(\rho+\rho^{\prime}\right)}{\beta V_{0}}-\frac{\beta V_{0}}{\alpha V_{0}-\left(\rho+\rho^{\prime}\right)}\right],
\end{aligned}
$$

produces a density of energy for the holographic component

$$
\rho_{2}^{\mathrm{MHRDE}}=\frac{V_{0}}{\Delta}\left[A \alpha-A \frac{\beta \sqrt{1+a^{6}}}{a^{3}}+\frac{\beta(A-1)}{a^{3}} \sinh ^{-1}\left(a^{-3}\right)\right] .
$$

The corresponding global EoS,

$$
\omega=\frac{\alpha a^{3}-\beta \sqrt{1+a^{6}}}{-\beta \sinh ^{-1}\left(a^{-3}\right)-\alpha a^{3}+\beta \sqrt{1+a^{6}}},
$$

has asymptotic values $\omega_{e}=0$ for $a=0$ and $\omega_{l}=-1$ at late times. Nevertheless the maximum value of $\omega$ is positive in the intermediate epoch between the asymptotic dust era $a=0$ and the truly dust era $a=\beta^{1 / 3}(\alpha-\beta)^{-1 / 6}(\alpha+\beta)^{-1 / 6}$ because $g=(\alpha-$ $\beta \cosh (\sqrt{-x})) /(-2 \beta \sqrt{-x} \sinh (\sqrt{-x})$ is not always negative at early times. Note that the constants $\alpha$ and $\beta$ should be adjusted so that the holographic density is kept positive and the quotient of densities in the dark sector fits with the current value. The adiabatic velocity of sound $c_{\mathrm{s}}^{2}=\left(\sinh ^{-1}\left(a^{-3}\right) \sqrt{1+a^{6}}\right)^{-1}$ oscillates between $c_{\mathrm{s}}^{2}=0$ at early times and $c_{\mathrm{s}}^{2}=1$ at late times, independently from the constants $A, B, \alpha$, and $\beta$.

\section{Conclusions}

In this work we have studied cosmological models driven by k-essences with constant potential $V_{0}$, generated by strictly increasing $\left(F_{x}>0\right)$ and concave $\left(F_{x x}<0\right)$ kinetic functions $F$. The study is described in a FRW background and the goal was the possibility of finding links between these universes and interactive models filled with CDM and MHRDE fluids. This idea is supported in studies realized previously where scalar representations of cosmological interactive arbitrary systems were found using exotic quintessence [48] and exotic $\mathrm{k}$-essence as scalar fields [50]. Here we particularize the interactive model, considering it as integrated by $\mathrm{CDM}$ and the MHRDE fluid, whose defining parameters $A$ and $B$ mark limits on the used k-essences. According to the general method described in [50], the fields turn out to be common k-essences derived from a Lagrangian $\mathcal{L}=-V_{0} F$. This fact could allow us to consider this formalism as an indirect or covert Lagrangian description of a cosmological system with interactive dark energy [76]. In the k-essential approach of the cosmological models they have not allowed the crossing of the phantom divide $(\omega=-1)$. This is clearly seen in Fig. 1, where the global EoS $\omega=g /(1-g)$ is plotted as a function of an auxiliary magnitude $g=F / 2 x F_{x}$. There are two branches in the picture, one describing viable universes and the other corresponding to phantom universes, and the cosmological constant type behavior is the asymptotic conduct in the extreme points $\left(F_{x}=0\right)$, when we arrive at the low limit $g \rightarrow-\infty$ of the acceptable branch $g<1$ or at the top 
limit $g \rightarrow+\infty$ of the phantom branch $g>1$. Restricting ourselves to models with no phantom for which $g<1$, a positive global density of energy leads to the condition $F_{x}>0$, while the stability measured by the adiabatic speed of sound determines $F_{x x}<0$. These conditions select possible kessences for the representation $(F \rightarrow Q)$ and simultaneously they reject interactions that can lead, through this approach, to cosmologically nonviable systems $(Q \rightarrow F)$. Two other restrictions on these models of universe arising from the use of CDM and MHRDE as interacting fluids are the inability to have constant ratios $r=r_{0}$ to alleviate the problem of the coincidence without having a global constant $\operatorname{EoS} \omega=\omega_{0}$ (because $\left.r=r_{0}=\left(A-1-\omega_{0}\right) /\left(1+\omega_{0}-B\right)\right)$, and the existence of a maximum value for the global $\operatorname{EoS} \omega_{\max }=A-1$.

The link between both schemes arises from equalizing the expressions of the time derivative of the Hubble parameter, $-2 \dot{H}=(\rho+p)=-2 x F_{x} V_{0}$, in each description and from supposing a linear combination of density of energy and of pressure for the $D E$. Then the conservation equation for the $D E$ gives us the expression that must hold for the kinetic function $F$ and for the interaction $Q$. From (15), given the interaction $Q\left(V_{0}, F\right)$, finding the function $F$ allows one to write all the densities of energy as a function of the factor of scale through Eqs. (10), (12), and (13). Inversely, given the appropriate function $F$ we find the interaction that affects the CDM-MHRDE system. The last two sections were dedicated to giving examples of these two manners of using (15). With the CDM-MHRDE systems affected by the interactions $Q=0, Q_{\Lambda}=\left(B \rho+(1-\Delta) \rho^{\prime}\right) / \Delta$, and $Q_{\mathrm{sc}}=B \rho_{2}-\rho_{1}$ we associate systems driven by the k-essences $F(x)=\left(F_{0}+F_{1} \sqrt{-x}\right)^{B /(B-1)}, F=F_{1}+$ $2 F_{0} \frac{(1-A)}{A}(-x)^{\frac{A}{2(A-1)}}$, and $F^{ \pm}=F_{0}^{ \pm}(-x)^{y^{ \pm}}$, respectively, showing that they arrive at the same dynamic results in both approaches, but in a more direct way. Also, in the particular case $Q_{\Lambda}$ we show that the concept of a cosmological constant can be interpreted as the result of an interaction in these CDM-MHRDE systems. For the inverse way, we use the kinetic functions $F(x)=1+m x, F(x)=\frac{b}{6}+x-\frac{x^{2}}{2 b}$, and $F=\alpha-\beta \cosh (\sqrt{-x})$ to obtain interactions not usually considered in the literature, $Q=\left(B \rho+(B-1) \rho^{\prime}\right) / \Delta$, $Q=\left(B \rho+B \rho^{\prime}+\frac{\rho^{\prime \prime}}{4}+\frac{\rho^{\prime}\left(6 \rho / b V_{0}\right)^{-1 / 2}}{2}\right) / \Delta$, and $Q=$ $\left[B\left(\rho+\rho^{\prime}\right)+\beta V_{0}\left[\frac{\alpha V_{0}-\left(\rho+\rho^{\prime}\right)}{\beta V_{0}}-\frac{\beta V_{0}}{\alpha V_{0}-\left(\rho+\rho^{\prime}\right)}\right]\right] / \Delta$, respectively. The latter case shows the difference between the maximum value of the global EoS and its asymptotic limits. Moreover, in the last two cases, we obtained interactions that with difficulty could be solved by the method of the source equation and for which, nevertheless, we obtained explicit expressions as regards all the cosmological important magnitudes.

Open Access This article is distributed under the terms of the Creative Commons Attribution 4.0 International License (http://creativecomm ons.org/licenses/by/4.0/), which permits unrestricted use, distribution, and reproduction in any medium, provided you give appropriate credit to the original author(s) and the source, provide a link to the Creative Commons license, and indicate if changes were made.

Funded by SCOAP ${ }^{3}$.

\section{References}

1. A.G. Riess et al. [Supernova Search Team Collaboration], Astron. J. 116, 1009 (1998). doi:10.1086/300499. arXiv:astro-ph/9805201

2. S. Perlmutter et al. [Supernova Cosmology Project Collaboration], Astrophys. J. 517, 565 (1999). doi:10.1086/307221. arXiv:astro-ph/9812133

3. R. Amanullah et al., Astrophys. J. 716, 712 (2010). doi:10.1088/ 0004-637X/716/1/712. arXiv:1004.1711 [astro-ph.CO]

4. D.N. Spergel et al. [WMAP Collaboration], Astrophys. J. Suppl. 170, 377 (2007). doi:10.1086/513700. arXiv:astro-ph/0603449

5. D.H. Weinberg, M.J. Mortonson, D.J. Eisenstein, C. Hirata, A.G. Riess, E. Rozo, Phys. Rep. 530, 87 (2013). doi:10.1016/j.physrep. 2013.05.001. arXiv:1201.2434 [astro-ph.CO]

6. W.J. Percival, S. Cole, D.J. Eisenstein, R.C. Nichol, J.A. Peacock, A.C. Pope, A.S. Szalay, Mon. Not. R. Astron. Soc. 381, 1053 (2007). doi:10.1111/j.1365-2966.2007.12268.x. arXiv:0705.3323 [astro-ph]

7. I. Zlatev, L.M. Wang, P.J. Steinhardt, Phys. Rev. Lett. 82, 896 (1999). doi:10.1103/PhysRevLett.82.896. arXiv:astro-ph/9807002

8. R.R. Caldwell, R. Dave, P.J. Steinhardt, Phys. Rev. Lett. 80, 1582 (1998). doi:10.1103/PhysRevLett.80.1582. arXiv:astro-ph/9708069

9. J.A. Frieman, C.T. Hill, A. Stebbins, I. Waga, Phys. Rev. Lett. 75, 2077 (1995). doi:10.1103/PhysRevLett.75.2077. arXiv:astro-ph/9505060

10. P.J.E. Peebles, B. Ratra, Astrophys. J. 325, L17 (1988). doi:10. $1086 / 185100$

11. B. Ratra, P.J.E. Peebles, Phys. Rev. D 37, 3406 (1988). doi:10. 1103/PhysRevD.37.3406

12. C. Armendariz-Picon, V.F. Mukhanov, P.J. Steinhardt, Phys. Rev. Lett. 85, 4438 (2000). doi:10.1103/PhysRevLett.85.4438. arXiv:astro-ph/0004134

13. C. Armendariz-Picon, V.F. Mukhanov, P.J. Steinhardt, Phys. Rev. D 63, 103510 (2001). doi:10.1103/PhysRevD.63.103510. arXiv:astro-ph/0006373

14. E.J. Copeland, M. Sami, S. Tsujikawa, Int. J. Mod. Phys. D 15, 1753 (2006). doi:10.1142/S021827180600942X. arXiv:hep-th/0603057

15. L.P. Chimento, Phys. Rev. D 69, 123517 (2004). doi:10.1103/ PhysRevD.69.123517. arXiv:astro-ph/0311613

16. T. Chiba, Phys. Rev. D 66, 063514 (2002). doi:10.1103/PhysRevD. 66.063514. arXiv:astro-ph/0206298

17. S. Tsujikawa, M. Sami, Phys. Lett. B 603, 113 (2004). doi:10.1016/ j.physletb.2004.10.023. arXiv:hep-th/0409212

18. M. Malquarti, E.J. Copeland, A.R. Liddle, M. Trodden, Phys. Rev. D 67, 123503 (2003). doi:10.1103/PhysRevD.67.123503. arXiv:astro-ph/0302279

19. J.M. Aguirregabiria, L.P. Chimento, R. Lazkoz, Phys. Rev. D 70, 023509 (2004). doi:10.1103/PhysRevD.70.023509. arXiv:astro-ph/0403157

20. L.P. Chimento, M.I. Forte, R. Lazkoz, Mod. Phys. Lett. A 20, 2075 (2005). doi:10.1142/S0217732305018074. arXiv:astro-ph/0407288

21. L.P. Chimento, M.I. Forte, Phys. Rev. D 73, 063502 (2006). doi:10. 1103/PhysRevD.73.063502. arXiv:astro-ph/0510726

22. C. Armendariz-Picon, E.A. Lim, JCAP 0508, 007 (2005). doi:10. 1088/1475-7516/2005/08/007. arXiv:astro-ph/0505207

23. M. Li, X. Zhang, Phys. Lett. B 573, 20 (2003). doi:10.1016/j. physletb.2003.08.041. arXiv:hep-ph/0209093 
24. Z.K. Guo, Y.S. Piao, X.M. Zhang, Y.Z. Zhang, Phys. Lett. B 608, 177 (2005). doi:10.1016/j.physletb.2005.01.017. arXiv:astro-ph/0410654

25. Y.F. Cai, E.N. Saridakis, M.R. Setare, J.Q. Xia, Phys. Rep. 493, 1 (2010). doi:10.1016/j.physrep.2010.04.001. arXiv:0909.2776 [hep-th]

26. B. Feng, X.L. Wang, X.M. Zhang, Phys. Lett. B 607, 35 (2005). doi:10.1016/j.physletb.2004.12.071. arXiv:astro-ph/0404224

27. B. Feng, M. Li, Y.S. Piao, X. Zhang, Phys. Lett. B 634, 101 (2006). doi:10.1016/j.physletb.2006.01.066. arXiv:astro-ph/0407432

28. L.P. Chimento, M.I. Forte, R. Lazkoz, M.G. Richarte, Phys. Rev. D 79, 043502 (2009). doi:10.1103/PhysRevD.79.043502. arXiv:0811.3643 [astro-ph]

29. G. 't Hooft, Salamfest, pp. 0284-296 (1993). arXiv:gr-qc/9310026

30. W. Fischler, L. Susskind, arXiv:hep-th/9806039

31. O. Aharony, S.S. Gubser, J.M. Maldacena, H. Ooguri, Y. Oz, Phys. Rep. 323, 183 (2000). doi:10.1016/S0370-1573(99)00083-6. arXiv:hep-th/9905111

32. R. Bousso, Rev. Mod. Phys. 74, 825 (2002). doi:10.1103/ RevModPhys.74.825. arXiv:hep-th/0203101

33. R. Bousso, JHEP 9907, 004 (1999). doi:10.1088/1126-6708/1999/ 07/004. arXiv:hep-th/9905177

34. S.D.H. Hsu, Phys. Lett. B 594, 13 (2004). doi:10.1016/j.physletb. 2004.05.020. arXiv:hep-th/0403052

35. L. Amendola, Phys. Rev. D 62, 043511 (2000). doi:10.1103/ PhysRevD.62.043511. arXiv:astro-ph/9908023

36. G. Mangano, G. Miele, V. Pettorino, Mod. Phys. Lett. A 18,831 (2003). doi:10.1142/S0217732303009940. arXiv:astro-ph/0212518

37. S. del Campo, R. Herrera, D. Pavon, Phys. Rev. D 70, $043540 \quad$ (2004). doi:10.1103/PhysRevD.70.043540. arXiv:astro-ph/0407047

38. G.R. Farrar, P.J.E. Peebles, Astrophys. J. 604, 1 (2004). doi:10. 1086/381728. arXiv:astro-ph/0307316

39. Z.K. Guo, R.G. Cai, Y.Z. Zhang, JCAP 0505, 002 (2005). doi:10. 1088/1475-7516/2005/05/002. arXiv:astro-ph/0412624

40. Z.K. Guo, Y.Z. Zhang, Phys. Rev. D 71, 023501 (2005). doi:10. 1103/PhysRevD.71.023501. arXiv:astro-ph/0411524

41. R.G. Cai, A. Wang, JCAP 0503, 002 (2005). doi:10.1088/ 1475-7516/2005/03/002. arXiv:hep-th/0411025

42. B. Gumjudpai, T. Naskar, M. Sami, S. Tsujikawa, JCAP 0506, 007 (2005). doi:10.1088/1475-7516/2005/06/007. arXiv:hep-th/0502191

43. R. Curbelo, T. Gonzalez, G. Leon, I. Quiros, Class. Quantum Gravity 23, 1585 (2006). doi:10.1088/0264-9381/23/5/010. arXiv:astro-ph/0502141

44. W. Zimdahl, D. Pavon, Phys. Lett. B 521, 133 (2001). doi:10.1016/ S0370-2693(01)01174-1. arXiv:astro-ph/0105479

45. L.P. Chimento, A.S. Jakubi, D. Pavon, W. Zimdahl, Phys. Rev. D 67, 083513 (2003). doi:10.1103/PhysRevD.67.083513. arXiv:astro-ph/0303145

46. G. Olivares, F. Atrio-Barandela, D. Pavon, Phys. Rev. D 71, 063523 (2005). doi:10.1103/PhysRevD.71.063523. arXiv:astro-ph/0503242

47. M. Szydlowski, Cosmological model with energy transfer. Phys. Lett. B 632, 1 (2006). doi:10.1016/j.physletb.2005.10.039. arXiv:astro-ph/0502034

48. L. Chimento, M.I. Forte, Phys. Lett. B 666, 205 (2008). doi:10. 1016/j.physletb.2008.07.064. arXiv:0706.4142 [astro-ph]
49. M. Forte, arXiv:1610.07441 [hep-th]

50. M. Forte, Eur. Phys. J. C 76(1), 42 (2016). doi:10.1140/epjc/ s10052-016-3882-6. arXiv:1507.03658 [gr-qc]

51. L.N. Granda, A. Oliveros, Phys. Lett. B 671, 199 (2009). doi:10. 1016/j.physletb.2008.12.025. arXiv:0810.3663 [gr-qc]

52. S. Nojiri, S.D. Odintsov, Gen. Relativ. Gravity 38, 1285 (2006) doi:10.1007/s10714-006-0301-6. arXiv:hep-th/0506212

53. M. Li, Phys. Lett. B 603, 1 (2004). doi:10.1016/j.physletb.2004. 10.014. arXiv:hep-th/0403127

54. L.P. Chimento, M.I. Forte, M.G. Richarte, Mod. Phys. Lett. A 28, 1250235 (2013). doi:10.1142/S0217732312502355. arXiv:1106.0781 [astro-ph.CO]

55. L.P. Chimento, M. Forte, M.G. Richarte, Eur. Phys. J. C 73(1), 2285 (2013). doi:10.1140/epjc/s10052-013-2285-1. arXiv:1301.2737 [gr-qc]

56. P. Pankunni, T.K. Mathew, Int. J. Mod. Phys. D 23, 1450024 (2014). doi:10.1142/S0218271814500242. arXiv:1309.3136 [astro-ph.CO]

57. E.K. Li, Y. Zhang, J.L. Geng, Phys. Rev. D 90(8), 083534 (2014). doi:10.1103/PhysRevD.90.083534. arXiv:1412.5482 [gr-qc]

58. A. Pasqua, S. Chattopadhyay, R. Myrzakulov, arXiv:1511.00600 [gr-qc]

59. M. Sharif, A. Jawad, Astrophys. Space Sci. 337, 789 (2012). doi:10. 1007/s10509-011-0893-5

60. S. del Campo, J.C. Fabris, R. Herrera, W. Zimdahl, Phys. Rev. D 83, 123006 (2011). doi:10.1103/PhysRevD.83.123006. arXiv:1103.3441 [astro-ph.CO]

61. R.C.G. Landim, Int. J. Mod. Phys. D 25(04), 1650050 (2016). doi:10.1142/S0218271816500504. arXiv:1508.07248 [hep-th]

62. K. Bamba, S. Capozziello, S. Nojiri, S.D. Odintsov, Astrophys. Space Sci. 342, 155 (2012). doi:10.1007/s10509-012-1181-8. arXiv:1205.3421 [gr-qc]

63. L.N. Granda, A. Oliveros, Phys. Lett. B 669, 275 (2008). doi:10. 1016/j.physletb.2008.10.017. arXiv:0810.3149 [gr-qc]

64. Y.B. Zeldovich, Mon. Not. R. Astron. Soc. 160, 1P (1972)

65. P.H. Chavanis, Phys. Rev. D 92(10), 103004 (2015). doi:10.1103/ PhysRevD.92.103004. arXiv:1412.0743 [gr-qc]

66. S.W. Hawking, G.F.R. Ellis, doi:10.1017/CBO9780511524646

67. R.M. Wald, (University of Chicago Press, USA, 1984), p. 491. doi:10.7208/chicago/9780226870373.001.0001. ISBN: 9780226870335

68. R. Bean, O. Dore, Phys. Rev. D 69, 083503 (2004). doi:10.1103/ PhysRevD.69.083503. arXiv:astro-ph/0307100

69. M.S. Longair, doi:10.1007/978-3-540-73478-9

70. T. Padmanabhan, (Cambridge University Press, Cambridge, UK, 2010). doi:10.1017/CBO9780511807787

71. P. Coles, F. Lucchin, (Wiley, Chichester, 2002), p. 492. ISBN: 9780-471-48909-2

72. J. Garriga, V.F. Mukhanov, Phys. Lett. B 458, 219 (1999). doi:10. 1016/S0370-2693(99)00602-4. arXiv:hep-th/9904176

73. E. Babichev, V. Mukhanov, A. Vikman, JHEP 0802, 101 (2008). doi:10.1088/1126-6708/2008/02/101. arXiv:0708.0561 [hep-th]

74. A. Vikman, Phys. Rev. D 71, 023515 (2005). doi:10.1103/ PhysRevD.71.023515. arXiv:astro-ph/0407107

75. L.P. Chimento, Phys. Rev. D 81, 043525 (2010). doi:10.1103/ PhysRevD.81.043525. arXiv:0911.5687 [astro-ph.CO]

76. N.J. Poplawski, arXiv:gr-qc/0608031 